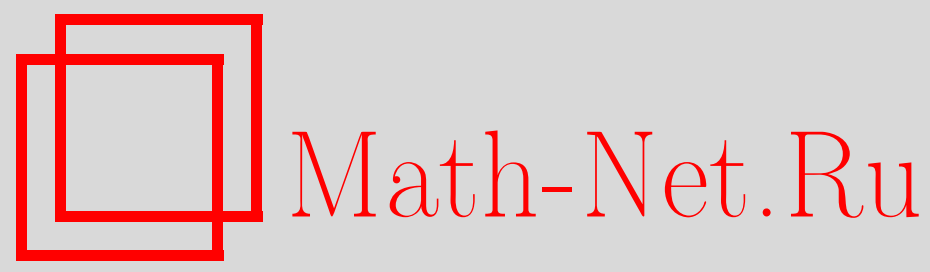

В. А. Краснов, Группа Брауэра и группа Витта вещественной линейчатой поверхности, Матем. заметки, 2002, том 72, выпуск 5, 706-714

DOI: https://doi.org/10.4213/mzm459

Использование Общероссийского математического портала Math-Net.Ru подразумевает, что вы прочитали и согласны с пользовательским соглашением http://www . mathnet.ru/rus/agreement

Параметры загрузки:

IP : 44.207 .124 .84

26 апреля 2023 г., 13:17:01

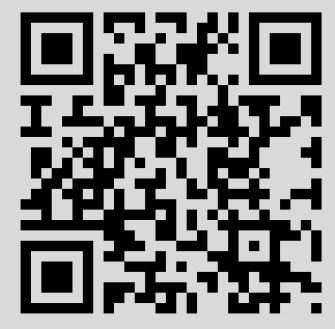


том 72 выпУск 5 нояБрь 2002

УДК 513.6

\title{
ГРУППА БРАУЭРА И ГРУППА ВИТТА ВЕЩЕСТВЕННОЙ ЛИНЕЙЧАТОЙ ПОВЕРХНОСТИ
}

\author{
В. А. Краснов
}

Вычисляется группа Брауэра и группа Витта вещественной иррациональной линейчатой поверхности. Вычисления производятся с помощью эквивариантных когомологий.

Библиография: 9 названий.

1. Введение. Далее $X$ - неособая вешественная проективная поверхность такая, что $X_{\mathbb{C}}=X \otimes \mathbb{C}$ - иррациональная поверхность, т.е. $X_{\mathbb{C}}$ бирационально эквивалентна прямому произведению алгебраической кривой рода $q>0$ на проективную прямую. Будем предполагать, что $X(\mathbb{R}) \neq \varnothing$. Тогда зафиксируем точку $x_{0} \in X(\mathbb{R})$ и рассмотрим соответствующее отображение Альбанезе $\mu: X \rightarrow A$. Образ $\mu(X)$ является неособой кривой рода $q$; обозначим ее через $E$ и рассмотрим отображение $\mu: X \rightarrow E$. Заметим, что отображение вещественных точек $\mu: X(\mathbb{R}) \rightarrow E(\mathbb{R})$ может быть несюръективньм. Пусть $E_{1}, \ldots, E_{r}$ - компоненты связности множества $E(\mathbb{R})$; тогда благодаря отображению $\mu: X(\mathbb{R}) \rightarrow E(\mathbb{R})$ они разбиваются на следующие три класса. Компонента $E_{i}$ принадлежит первому классу, если $E_{i} \subset \mu(X(\mathbb{R}))$. Пусть множество $\mu(X(\mathbb{R}))$ не содержит $E_{i}$, но пересечение $E_{i} \cap \mu(X(\mathbb{R}))$ непустое; тогда по определению компонента $E_{i}$ принадлежит второму классу. Наконец, если $E_{i} \cap \mu(X(\mathbb{R}))=\varnothing$, то $E_{i}$ принадлежит третьему классу. Через $r_{1}, r_{2}$ и $r_{3}$ будем обозначать число компонент множества $E(\mathbb{R})$, принадлежащих соответственно первому, второму и третьему классам, а через $s$ обозначим число компонент связности множества $X(\mathbb{R})$. Тогда справедливы следующие теоремы о групе Брауэра и группе Витта.

Теорема 1. Выполняется равенство

$$
\operatorname{Br}(X)=(\mathbb{Z} / 2)^{l},
$$

$2 \partial e$

$$
l= \begin{cases}2 s-r_{1}+r_{3}-1, & \text { если } r_{1}<r \\ s & \text { в противном случае. }\end{cases}
$$


Теорема 2. Выполняется равенство

$$
W(X)=\mathbb{Z}^{s} \oplus(\mathbb{Z} / 2)^{m} \oplus(\mathbb{Z} / 4)^{n},
$$

$2 \partial e$

$$
m=\left\{\begin{array}{ll}
q+s-r_{1}-1, & \text { если } r_{2}>0, \\
q+s-r_{1}+1, & \text { если } r_{2}=0, r_{3}>0, \\
q, & \text { если } r_{2}=0, r_{3}=0,
\end{array} \quad n= \begin{cases}r_{3}, & \text { если } r_{2}>0, \\
r_{3}-1, & \text { если } r_{2}=0, r_{3}>0 \\
0, & \text { если } r_{2}=0, r_{3}=0\end{cases}\right.
$$

2. Предварительные результаты. При стягивании 1-го рода $X \rightarrow X^{\prime}$ группы $\operatorname{Br}(X), W(X)$ не меняются, также не меняются инварианты $r_{1}, r_{2}, r_{3}, s$, поэтому мы можем считать поверхность $X$ относительно минимальной. В этом случае отображение $\mu: X(\mathbb{C}) \rightarrow E(\mathbb{C})$ не имеет особых слоев над комплексньми точками из $E(\mathbb{C})$, а каждый особый слой $C_{e}$ над вешественной точкой $e \in E(\mathbb{R})$ состоит из двух проективных прямых $C_{e}^{(1)}, C_{e}^{(2)}$ с индексами пересечения $\left(C_{e}^{(1)}\right)^{2}=\left(C_{e}^{(2)}\right)^{2}=-1, C_{e}^{(1)} \cdot C_{e}^{(2)}=1$, причем инволюция $g: X(\mathbb{C}) \rightarrow X(\mathbb{C})$ отображает прямую $C_{e}^{(1)}$ в прямую $C_{e}^{(2)}$. Заметим также, что каждьй особый слой $C_{e}$ находится над точкой из компоненты связности $E(\mathbb{R})$, принадлежащей второму классу. Обозначим через $\omega_{1}^{0}, \ldots, \omega_{r}^{0} \in H^{0}\left(E(\mathbb{R}), \mathbb{F}_{2}\right)$, $\omega_{1}^{1}, \ldots, \omega_{r}^{1} \in H^{1}\left(E(\mathbb{R}), \mathbb{F}_{2}\right)$ классы когомологий, определенные компонентами $E_{1}, \ldots, E_{r}$. Тогда получаем следующую переформулировку разбиения компонент $E_{1}, \ldots, E_{r}$ на классы. Компонента $E_{i}$ принадлежит первому классу, если $\mu^{*}\left(\omega_{i}^{1}\right) \neq 0$ в $H^{1}\left(X(\mathbb{R}), \mathbb{F}_{2}\right)$. Если $\mu^{*}\left(\omega_{i}^{1}\right)=0$, но $\mu^{*}\left(\omega_{i}^{0}\right) \neq 0$, то $E_{i}$ принадлежит второму классу. Наконец, если $\mu^{*}\left(\omega_{i}^{0}\right)=0$, то $E_{i}$ принадлежит третьему классу.

Группа Галуа $G=G(\mathbb{C} / \mathbb{R})$ действует на $X(\mathbb{C}), E(\mathbb{C})$. Положим $Y=X(\mathbb{C}) / G$, $F=E(\mathbb{C}) / G$, и пусть $\pi: X(\mathbb{C}) \rightarrow Y, \pi: E(\mathbb{C}) \rightarrow F$-проекции. Отображение $\mu: X(\mathbb{C}) \rightarrow$ $E(\mathbb{C})$ индуцирует отображение $\mu: Y \rightarrow F$.

Лемма 1. Гомоморфизм

$$
\mu^{*}: H^{1}\left(F, \mathbb{F}_{2}\right) \rightarrow H^{1}\left(Y, \mathbb{F}_{2}\right)
$$

является мономорфизмом, размерность коядра которого равна $r_{3}$.

ДоказАтЕЛЬСтво. Рассмотрим коммутативную диаграмму

$$
\begin{aligned}
& 0 \longrightarrow H^{1}\left(F, \mathbb{F}_{2}\right) \longrightarrow H^{1}\left(E(\mathbb{C}) ; G, \mathbb{F}_{2}\right) \longrightarrow H^{0}\left(E(\mathbb{R}), \mathbb{F}_{2}\right) \longrightarrow 0 \\
& \downarrow \mu^{*} \quad \cong \downarrow \mu^{*} \quad \downarrow \mu^{*} \quad, \\
& 0 \longrightarrow H^{1}\left(Y, \mathbb{F}_{2}\right) \longrightarrow H^{1}\left(X(\mathbb{C}) ; G, \mathbb{F}_{2}\right) \longrightarrow H^{0}\left(X(\mathbb{R}), \mathbb{F}_{2}\right)
\end{aligned}
$$

строки которой точны. Эта диаграмма получается из первой спектральной последовательности для эквивариантных когомологий (см. подробности в [1]). Так как гомоморфизм

$$
\mu^{*}: H^{k}\left(E(\mathbb{C}), \mathbb{F}_{2}\right) \rightarrow H^{k}\left(X(\mathbb{C}), \mathbb{F}_{2}\right)
$$


является изоморфизмом при $k=0,1$, то средний гомоморфизм $\mu^{*}$ в диаграмме (1) является также изоморфизмом. Это утверждение вытекает из рассмотрения второй спектральной последовательности для эквивариантных когомологий. После этих замечаний утверждение леммы следует из диаграммы (1). Лемма доказана.

Множество $X(\mathbb{R})$ можно рассматривать вложенным в $Y$, поэтому определен гомоморфизм ограничения

$$
\beta_{Y}: H^{1}\left(Y, \mathbb{F}_{2}\right) \rightarrow H^{1}\left(X(\mathbb{R}), \mathbb{F}_{2}\right)
$$

Размерность ядра этого гомоморфизма обозначим через $k^{1}(Y)$.

\section{ЛЕмма 2. Выполняется равенство}

$$
k^{1}(Y)= \begin{cases}q-r_{1}+r_{3}, & \text { если } r_{1}<r \\ q-r+1, & \text { если } r_{1}=r .\end{cases}
$$

ДоКАЗАТЕЛЬСТВо. Гомоморфизм ограничения $\beta_{Y}$ раскладьвается в композицию гомоморфизмов

$$
\begin{aligned}
H^{1}\left(Y, \mathbb{F}_{2}\right) \stackrel{\pi^{*}}{\longrightarrow}\left(H^{1}\left(X(\mathbb{C}), \mathbb{F}_{2}\right)\right)^{G} \stackrel{\left(\mu^{*}\right)^{-1}}{\longrightarrow}\left(H^{1}\left(E(\mathbb{C}), \mathbb{F}_{2}\right)\right)^{G} \\
\stackrel{\beta_{E}}{\longrightarrow} H^{1}\left(E(\mathbb{R}), \mathbb{F}_{2}\right) \stackrel{\mu^{*}}{\longrightarrow} H^{1}\left(X(\mathbb{R}), \mathbb{F}_{2}\right),
\end{aligned}
$$

причем $\pi^{*}$ - мономорфизм. Рассмотрим гомоморфизмы ограничения

$$
\beta_{F}: H^{1}\left(F, \mathbb{F}_{2}\right) \rightarrow H^{1}\left(E(\mathbb{R}), \mathbb{F}_{2}\right), \quad \beta_{E}: H^{1}\left(E(\mathbb{C}), \mathbb{F}_{2}\right)^{G} \rightarrow H^{1}\left(E(\mathbb{R}), \mathbb{F}_{2}\right) ;
$$

их образы совпадают и равны гиперплоскости $[E(\mathbb{R})]^{\perp}$, состоящей из элементов $a \in$ $H^{1}\left(E(\mathbb{R}), \mathbb{F}_{2}\right)$ таких, что $a([E(\mathbb{R})])=0$. Пусть $k^{1}(F)$ - размерность ядра гомоморфизма $\beta_{F}$. Тогда $k^{1}(F)=q-r+1$. Из леммы 1 и коммутативной диаграммы

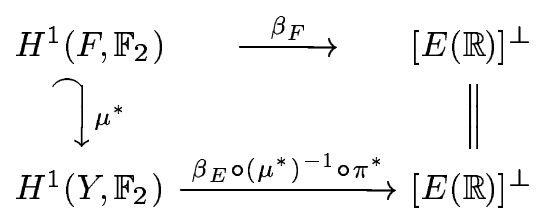

следует, что ядро гомоморфизма $\beta_{E} \circ\left(\mu^{*}\right)^{-1} \circ \pi^{*}$ имеет размерность $q-r+r_{3}+1$. Так как в диаграмме (3) строки являются эпиморфизмами, из разложения (2) и описания гомоморфизма

$$
\mu^{*}: H^{1}\left(E(\mathbb{R}), \mathbb{F}_{2}\right) \rightarrow H^{1}\left(X(\mathbb{R}), \mathbb{F}_{2}\right)
$$

следует утверждение леммы о $k^{1}(Y)$. Лемма доказана.

Пусть $e \in E(\mathbb{R})$ и $C_{e}$ - слой отображения $\mu: X(\mathbb{C}) \rightarrow E(\mathbb{C})$ над точкой $e$. Класс гомологий $\left[C_{e}\right] \in H_{2}(X(\mathbb{C}), \mathbb{Z})$ не зависит от выбора точки $e$, обозначим его через $c$. Если $g: X(\mathbb{C}) \rightarrow X(\mathbb{C})$ - инволюция комплексного сопряжения, то $g_{*}(c)=-c$, так как отображение $g: C_{e} \rightarrow C_{e}$ меняет ориентацию. Поэтому элемент $c \in H_{2}(X(\mathbb{C}), \mathbb{Z})$ определяет класс когомологий $[c] \in H^{1}\left(G, H_{2}(X(\mathbb{C}), \mathbb{Z})\right)$. 
ЛЕмма 3. Если $r_{2}>0$, то $[c]=0$, в противном случае $[c] \neq 0$.

ДокАЗАтЕльство. Если $r_{2}>0$, то существует точка $e \in E(\mathbb{R})$ такая, что слой $C_{e}$ распадается на комплексные проективные прямые $C_{e}^{(1)}, C_{e}^{(2)}$. Tак как $g_{*}\left(\left[C_{e}^{(1)}\right]\right)=$ $-\left[C_{e}^{(2)}\right]$, имеем равенство

$$
\left[C_{e}\right]=\left[C_{e}^{(1)}\right]-g_{*}\left(\left[C_{e}^{(1)}\right]\right)
$$

Поэтому класс $[c]$ равен нулю в $H^{1}\left(G, H_{2}(X(\mathbb{C}), \mathbb{Z})\right)$. Пусть теперь $r_{2}=0$. Тогда расслоение $\mu: X(\mathbb{C}) \rightarrow E(\mathbb{C})$ является косьм произведением со слоем комплексная проективная прямая, поэтому

$$
H_{2}(X(\mathbb{C}), \mathbb{Z})=\mathbb{Z}^{2},
$$

причем класс $c \in H_{2}(X(\mathbb{C}), \mathbb{Z})$ будет одной из образуюших этой группы. Положим $E^{(1)}=$ $\mu(X(\mathbb{R}))$. Тогда $E^{(1)}$ состоит из компонент первого класса и отображение $\mu: X(\mathbb{R}) \rightarrow$ $E^{(1)}$ является косьм произведением со слоем окружность; следовательно, $\chi(X(\mathbb{R}))=0$. $\mathrm{C}$ другой стороны, из формулы Лефшеца о неподвижных точках имеем равенство

$$
\chi(X(\mathbb{R}))=2+\operatorname{rk} H_{2}(X(\mathbb{C}), \mathbb{Z})_{+}-\operatorname{rk} H_{2}(X(\mathbb{C}), \mathbb{Z})_{-},
$$

где $H_{2}(X(\mathbb{C}), \mathbb{Z})_{ \pm}$- инвариантная и антиинвариантная подгрупш относительно инволюции $g_{*}: H_{2}(X(\mathbb{C}), \mathbb{Z}) \rightarrow H_{2}(X(\mathbb{C}), \mathbb{Z})$. Так как rk $H_{2}(X(\mathbb{C}), \mathbb{Z})=2$ и $\chi(X(\mathbb{R}))=0$, из равенства $(4)$ следует, что $H_{2}(X(\mathbb{C}), \mathbb{Z})_{-}=H_{2}(X(\mathbb{C}), \mathbb{Z})$, поэтому

$$
H^{1}\left(G, H_{2}(X(\mathbb{C}), \mathbb{Z})\right)=H_{2}(X(\mathbb{C}), \mathbb{Z}) / 2 .
$$

Следовательно, класс $[c]$ не равен нулю. Лемма доказана.

Далее мы будем рассматривать эквивариантные гомологии $H_{k}(X(\mathbb{C}) ; G, \mathbb{Z})$. Они определяются как полные когомологии бикомплекса (см. [2], [3]). А именно, если $C_{i}-$ группа цепей с целыми коэффициентами для некоторого эквивариантного клеточного разбиения $X(\mathbb{C})$, то

$$
H_{k}(X(\mathbb{C}) ; G, \mathbb{Z})=\frac{\operatorname{Ker}\left[d: \bigoplus_{i \geqslant k} C_{i} \rightarrow \bigoplus_{i \geqslant k-1} C_{i}\right]}{\operatorname{Im}\left[d: \bigoplus_{i \geqslant k+1} C_{i} \rightarrow \bigoplus_{i \geqslant k} C_{i}\right]},
$$

где $d$ - дифференциал, возникающий из бикомплекса

$$
\begin{aligned}
& \begin{array}{lllll}
\vdots & \vdots & \vdots & \vdots & \vdots
\end{array} \\
& \uparrow 1-g \quad \uparrow 1-g \quad \uparrow 1-g \quad \uparrow 1-g \quad \uparrow 1-g \\
& 0 \longrightarrow C_{4} \stackrel{\partial}{\longrightarrow} C_{3} \stackrel{\partial}{\longrightarrow} C_{2} \stackrel{\partial}{\longrightarrow} C_{1} \stackrel{\partial}{\longrightarrow} C_{0} \longrightarrow 0 \\
& \uparrow 1+g \quad \uparrow 1+g \quad \uparrow 1+g \quad \uparrow 1+g \quad \uparrow 1+g \\
& 0 \longrightarrow C_{4} \stackrel{\partial}{\longrightarrow} C_{3} \stackrel{\partial}{\longrightarrow} C_{2} \stackrel{\partial}{\longrightarrow} C_{1} \stackrel{\partial}{\longrightarrow} C_{0} \longrightarrow 0 \text {. } \\
& \uparrow 1-g \quad \uparrow 1-g \quad \uparrow 1-g \quad \uparrow 1-g \quad \uparrow 1-g \\
& 0 \longrightarrow C_{4} \stackrel{\partial}{\longrightarrow} C_{3} \stackrel{\partial}{\longrightarrow} C_{2} \stackrel{\partial}{\longrightarrow} C_{1} \stackrel{\partial}{\longrightarrow} C_{0} \longrightarrow 0 \\
& \uparrow \uparrow \uparrow \uparrow \uparrow \\
& \begin{array}{lllll}
0 & 0 & 0 & 0 & 0
\end{array}
\end{aligned}
$$


Рассмотрим вторую спектральную последовательность

$$
\mathrm{II}_{i, j}^{2}=H^{-i}\left(G, H_{j}(X(\mathbb{C}), \mathbb{Z})\right) \Longrightarrow H_{i+j}(X(\mathbb{C}) ; G, \mathbb{Z}) .
$$

Нас интересует дифференциал

$$
d_{-1,1}^{2}: H^{1}\left(G, H_{1}(X(\mathbb{C}), \mathbb{Z})\right) \rightarrow H^{3}\left(G, H_{2}(X(\mathbb{C}), \mathbb{Z})\right) .
$$

ЛЕмма 4. Дифференциал (5) равен нулю, если $r_{2}>0$ или $r_{1}=r$, в противном случае образ дифференциала (5) порожден классом $[c] \in H^{3}\left(G, H_{2}(X(\mathbb{C}), \mathbb{Z})\right)$.

ДокАЗАТЕЛЬСтво. Заметим, что

$$
H^{3}\left(G, H_{2}(X(\mathbb{C}), \mathbb{Z})\right)=H^{1}\left(G, H_{2}(X(\mathbb{C}), \mathbb{Z})\right),
$$

поэтому класс $[c]$ принадлежит $H^{3}\left(G, H_{2}(X(\mathbb{C}), \mathbb{Z})\right)$. В каждой компоненте $E_{i}$ выберем по точке $e_{i}$, причем предполагаем, что слой над ней неособый. Соединим точку $e_{i} \mathrm{c}$ точкой $e_{j}$ кривой $\Gamma_{i j}$ в $E(\mathbb{C}) \backslash E(\mathbb{R})$, где $i<j$. Тогда замкнутые кривые $\Gamma_{i j}-g\left(\Gamma_{i j}\right)$ порождают группу $H_{1}(E(\mathbb{C}), \mathbb{Z})_{-}$. Пусть $\varphi_{i j}: S \rightarrow E(\mathbb{C})$ - отображение окружности, которое определяет кривую $\Gamma_{i j}-g\left(\Gamma_{i j}\right), \mu: K_{i j} \rightarrow S$ - индуцированное расслоение со слоем комплексная проективная прямая. Тогда имеем коммутативную диаграмму

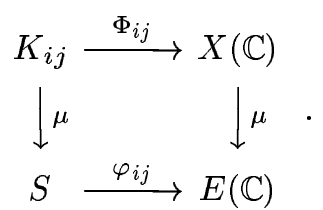

На расслоении $\mu: K_{i j} \rightarrow S$ действует инволюция $g: K_{i j} \rightarrow K_{i j}$, которая может быть трех видов: множество $\left(K_{i j}\right)^{G}$ состоит из двух окружностей, или из одной окружности, или пустое. Рассмотрим дифференциал

$$
d_{-1,1}^{2}: H^{1}\left(G, H_{1}\left(K_{i j}, \mathbb{Z}\right)\right) \rightarrow H^{3}\left(G, H_{2}\left(K_{i j}, \mathbb{Z}\right)\right) .
$$

Заметим, что расслоение $\mu: K_{i j} \rightarrow S$ тривиальное и

$$
H^{1}\left(G, H_{1}\left(K_{i j}, \mathbb{Z}\right)\right)=\mathbb{Z} / 2, \quad H^{3}\left(G, H_{2}\left(K_{i j}, \mathbb{Z}\right)\right)=\mathbb{Z} / 2 .
$$

Непосредственно проверяется, что дифференциал (6) ненулевой только для расслоения $K_{i j}$ второго вида, т.е. когда множество $\left(K_{i j}\right)^{G}$ состоит из одной окружности. Рассмотрим теперь следующую коммутативную диаграмму:

$$
\begin{aligned}
& \bigoplus_{i<j} H^{1}\left(G, H_{1}\left(K_{i j}, \mathbb{Z}\right)\right) \stackrel{d_{-1,1}^{2}}{\longrightarrow} \bigoplus_{i<j} H^{3}\left(G, H_{2}\left(K_{i j}, \mathbb{Z}\right)\right) \\
& \downarrow \Phi_{*} \\
& H^{1}\left(G, H_{1}(X(\mathbb{C}), \mathbb{Z})\right) \quad \stackrel{d_{-1,1}^{2}}{\longrightarrow} \quad H^{3}\left(G, H_{2}(X(\mathbb{C}), \mathbb{Z})\right)
\end{aligned}
$$

где гомоморфизмы $\Phi_{*}$ индуцированы эквивариантньми отображениями $\Phi_{i j}: K_{i j} \rightarrow$ $X(\mathbb{C})$. Тогда имеем равенства

$$
\begin{gathered}
\Phi_{*}\left(\bigoplus_{i<j} H^{1}\left(G, H_{1}\left(K_{i j}, \mathbb{Z}\right)\right)\right)=H^{1}\left(G, H_{1}(X(\mathbb{C}), \mathbb{Z})\right) \\
\Phi_{*}\left(H^{3}\left(G, H_{2}\left(K_{i j}, \mathbb{Z}\right)\right)\right)=(\mathbb{Z} / 2) \cdot[c]
\end{gathered}
$$

поэтому утверждение леммы 4 следует из описания дифференциала (6) и леммы 3 . Лемма доказана. 


\section{3. Доказательство теорем.}

ДокАЗАТЕЛЬСтво ТЕоремЫ 1. Так как для нашей поверхности $H^{2}(X(\mathbb{C}), \mathscr{O})=0$, то имеем равенство (см. [4]) $\operatorname{Br}(X)=H^{3}(X(\mathbb{C}) ; G, \mathbb{Z}-)_{\text {tors }}$, где $\mathbb{Z} \_-G$-модуль $\mathbb{Z}$, на котором инволюция $g$ действует умножением на -1 , а $H^{3}(X(\mathbb{C}) ; G, \mathbb{Z}-)$ tors - подгруппа кручения групшы эквивариантных когомологий. Групша $H^{3}(X(\mathbb{C}), \mathbb{Z})$ свободная, поэтому група $H^{3}(X(\mathbb{C}) ; G, \mathbb{Z}-$ ) tors 2 -периодическая (см. [4]). С другой стороны, в [5] для 2 -периодической подгрупшы $2 \operatorname{Br}(X)$ группы Брауэра установлено равенство

$$
\operatorname{dim}_{2} \operatorname{Br}(X)=\rho_{0}(X)-q(X)+k^{1}(Y)+2 s-1,
$$

где $\rho_{0}(X)$ - число Лефшеца; для нашей поверхности оно равно нулю, так как $H^{2}(X(\mathbb{C})$, $\mathscr{O})=0($ см. [4]). Следовательно,

$$
\operatorname{Br}(X)=(\mathbb{Z} / 2)^{l},
$$

где $l=2 s-1+k^{1}(Y)-q(X)$. Применим лемму 2 и заметим, что при $r_{1}=r$ имеем равенство $r=s$. Теорема 1 доказана.

ДокАЗАТЕЛЬСтво тЕоремЫ 2. Структура группы Витта вещественной проективной поверхности найдена в [6], а в работе [7] показано, как находить инварианты, с помощью которых описьвается групша $W(X)$ в [6]. Пусть

$$
\begin{aligned}
& k_{12}=\operatorname{dim} F^{1} H_{\mathrm{alg}}^{2}\left(X(\mathbb{C}) ; G, \mathbb{F}_{2}\right)+\operatorname{dim}\left(1+g^{*}\right) H^{1}\left(X(\mathbb{C}), \mathbb{F}_{2}\right) \\
& k_{13}=\operatorname{dim} H^{1}\left(Y, \mathbb{F}_{2}\right) \\
& k_{23}=\rho_{0}(X)-q(X)+k^{1}(Y)+s-1
\end{aligned}
$$

где пространство $F^{1} H_{\mathrm{alg}}^{2}\left(X(\mathbb{C}) ; G, \mathbb{F}_{2}\right)$ равно пересечению образа отображения цикла

$$
\operatorname{cl}: \operatorname{Pic} X \rightarrow H^{2}\left(X(\mathbb{C}) ; G, \mathbb{F}_{2}\right)
$$

с ядром гомоморфизма забывания вещественной структуры

$$
\alpha: H^{2}\left(X(\mathbb{C}) ; G, \mathbb{F}_{2}\right) \rightarrow H^{2}\left(X(\mathbb{C}), \mathbb{F}_{2}\right)
$$

Тогда выполняется равенство

$$
W(X)=\mathbb{Z}^{s} \oplus(\mathbb{Z} / 2)^{m} \oplus(\mathbb{Z} / 4)^{n},
$$

где $m=2 k_{12}-k_{13}+k_{23}, n=k_{13}-k_{12}$. Заметим, что

$$
\operatorname{dim}\left(1+g^{*}\right) H^{1}\left(X(\mathbb{C}), \mathbb{F}_{2}\right)=\operatorname{dim}\left(1+g^{*}\right) H^{1}\left(E(\mathbb{C}), \mathbb{F}_{2}\right)=q-r+1
$$

Из леммы 1 имеем

$$
\operatorname{dim} H^{1}\left(Y, \mathbb{F}_{2}\right)=\operatorname{dim} H^{1}\left(F, \mathbb{F}_{2}\right)=q+r_{3},
$$

поэтому

$$
k_{13}=q+r_{3}
$$


А из леммы 2 получаем

$$
k_{23}= \begin{cases}s-r_{1}+r_{3}-1, & \text { если } r_{1}<r, \\ 0 & \text { в противном случае. }\end{cases}
$$

Таким образом, остается вычислить

$$
\operatorname{dim} F^{1} H_{\mathrm{alg}}^{2}\left(X(\mathbb{C}) ; G, \mathbb{F}_{2}\right)
$$

Отображение цикла (7) раскладывается в композицию

$$
\operatorname{Pic} X \stackrel{\mathrm{cl}}{\longrightarrow} H^{2}\left(X(\mathbb{C}) ; G, \mathbb{Z}_{-}\right) \stackrel{R}{\longrightarrow} H^{2}\left(X(\mathbb{C}) ; G, \mathbb{F}_{2}\right),
$$

где $R$ - гомоморфизм редукции. Из равенства $H^{2}(X(\mathbb{C}), \mathscr{O})=0$ следует, что гомоморфизм

$$
\operatorname{cl}: \operatorname{Pic} X \rightarrow H^{2}\left(X(\mathbb{C}) ; G, \mathbb{Z}_{-}\right)
$$

эпиморфен (см. [8]). Следовательно, образ отображения цикла (7) равен образу гомоморфизма редукции

$$
R: H^{2}(X(\mathbb{C}) ; G, \mathbb{Z}-) \rightarrow H^{2}\left(X(\mathbb{C}) ; G, \mathbb{F}_{2}\right)
$$

Пусть $F^{1} H^{2}\left(X(\mathbb{C}) ; G, \mathbb{F}_{2}\right)$ - ядро гомоморфизма $(8)$, а $F^{1} H^{2}(X(\mathbb{C}) ; G, \mathbb{Z}-)$ - ядро гомоморфизма

$$
\alpha: H^{2}\left(X(\mathbb{C}) ; G, \mathbb{Z}_{-}\right) \rightarrow H^{2}\left(X(\mathbb{C}), \mathbb{Z}_{-}\right)
$$

Тогда имеем коммутативную диаграмму

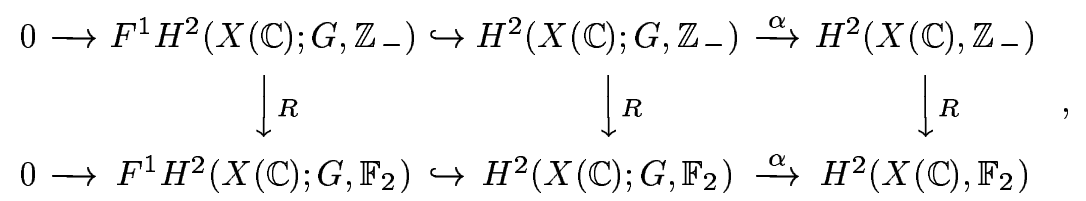

строки которой точные. Так как группа $H^{2}(X(\mathbb{C}), \mathbb{Z})$ свободная, левьй гомоморфизм $R$ в диаграмме (12) мономорфен. Поэтому

$\operatorname{dim} R\left(F^{1} H^{2}\left(X(\mathbb{C}) ; G, \mathbb{Z}_{-}\right)\right)=\operatorname{dim} F^{1} H^{2}\left(X(\mathbb{C}) ; G, \mathbb{Z}_{-}\right)=\operatorname{dim} H^{1}\left(G, H^{1}\left(X(\mathbb{C}), \mathbb{Z}_{-}\right)\right)$, где второе равенство следует из второй спектральной последовательности. Из равенств

$$
\operatorname{dim} H^{1}\left(G, H^{1}\left(X(\mathbb{C}), \mathbb{Z}_{-}\right)\right)=\operatorname{dim} H^{1}\left(G, H^{1}\left(E(\mathbb{C}), \mathbb{Z}_{-}\right)\right)=r-1
$$

получаем равенство

$$
R\left(F^{1} H^{2}\left(X(\mathbb{C}) ; G, \mathbb{Z}_{-}\right)\right)=r-1
$$

Рассмотрим группу

$$
\alpha^{-1}\left(2 H^{2}\left(X(\mathbb{C}), \mathbb{Z}_{-}\right)\right) / 2 H^{2}\left(X(\mathbb{C}) ; G, \mathbb{Z}_{-}\right)+F^{1} H^{2}(X(\mathbb{C}) ; G, \mathbb{Z}-)
$$


Гомоморфизм редукции $R$ отображает ее изоморфно на группу

$$
F^{1} H_{\mathrm{alg}}^{2}\left(X(\mathbb{C}) ; G, \mathbb{F}_{2}\right) / R\left(F^{1} H^{2}\left(X(\mathbb{C}) ; G, \mathbb{Z}_{-}\right)\right),
$$

а гомоморфизм $\alpha$ отображает изоморфно на группу

$$
\alpha\left(H^{2}\left(X(\mathbb{C}) ; G, \mathbb{Z}_{-}\right)\right) \cap 2 H^{2}\left(X(\mathbb{C}), \mathbb{Z}_{-}\right) / 2 \alpha\left(H^{2}\left(X(\mathbb{C}) ; G, \mathbb{Z}_{-}\right)\right)
$$

Группа $\alpha\left(H^{2}\left(X(\mathbb{C}) ; G, \mathbb{Z}_{-}\right)\right)$равна ядру дифференциала

$$
d_{2}^{0,2}: H^{2}\left(X(\mathbb{C}), \mathbb{Z}_{-}\right)^{G} \rightarrow H^{2}\left(G, H^{1}\left(X(\mathbb{C}), \mathbb{Z}_{-}\right)\right),
$$

а группа $\operatorname{Ker} d_{2}^{0,2} \cap 2 H^{2}\left(X(\mathbb{C}), \mathbb{Z}_{-}\right)$равна группе $2\left(H^{2}\left(X(\mathbb{C}), \mathbb{Z}_{-}\right)^{G}\right)$. Таким образом, группа (14) изоморфна групше

$$
2\left(H^{2}\left(X(\mathbb{C}), \mathbb{Z}_{-}\right)^{G}\right) / 2 \operatorname{Ker} d_{2}^{0,2}=H^{2}\left(X(\mathbb{C}), \mathbb{Z}_{-}\right)^{G} / \operatorname{Ker} d_{2}^{0,2} .
$$

Применим равенство (13). Тогда получим

$$
\operatorname{dim} F^{1} H_{\mathrm{alg}}^{2}\left(X(\mathbb{C}) ; G, \mathbb{F}_{2}\right)=\operatorname{dim}\left(H^{2}\left(X(\mathbb{C}), \mathbb{Z}_{-}\right)^{G} / \operatorname{Ker} d_{2}^{0,2}\right)+r+1 .
$$

Теперь мы хотим показать, что из леммы 4 следует равенство

$$
\operatorname{dim}\left(H^{2}\left(X(\mathbb{C}), \mathbb{Z}_{-}\right)^{G} / \operatorname{Ker} d_{2}^{0,2}\right)= \begin{cases}0, & \text { если } r_{2}>0 \text { или } r_{1}=r \\ 1 & \text { в противном случае. }\end{cases}
$$

Прежде всего заметим, что равенство (16) равносильно равенству

$$
\operatorname{dim}\left(\mathrm{II}_{2}^{1,2}(\mathbb{Z}) / \operatorname{Ker} d_{2}^{1,2}(\mathbb{Z})\right)= \begin{cases}0, & \text { если } r_{2}>0 \text { или } r_{1}=r, \\ 1 & \text { в противном случае, }\end{cases}
$$

где $\mathrm{II}_{2}^{1,2}(\mathbb{Z})=H^{1}\left(G, H^{2}(X(\mathbb{C}), \mathbb{Z})\right)$, a $d_{2}^{1,2}(\mathbb{Z})$ - дифференциал из спектральной последовательности для эквивариантных когомологий с коэффициентами в $\mathbb{Z}$. Применим $\cup$-произведение. Тогда получим, что равенство (17) равносильно равенству (см. [9])

$$
\operatorname{dim} \operatorname{Im} d_{2}^{1,3}(\mathbb{Z})= \begin{cases}0, & \text { если } r_{2}>0 \text { или } r_{1}=r \\ 1 & \text { в противном случае. }\end{cases}
$$

Применим двойственность Пуанкаре для эквивариантных (ко)гомологий. Тогда получим, что равенство (18) равносильно лемме 4. Итак, равенство (16) установлено, поэтому соотношение (15) дает равенство

$$
\operatorname{dim} F^{1} H_{\mathrm{alg}}^{2}\left(X(\mathbb{C}) ; G, \mathbb{F}_{2}\right)= \begin{cases}r-1, & \text { если } r_{2}>0 \text { или } r_{1}=r, \\ r & \text { в противном случае. }\end{cases}
$$

Применим равенство (9). Получим

$$
k_{12}= \begin{cases}q, & \text { если } r_{2}>0 \text { или } r_{1}=r \\ q+1 & \text { в противном случае. }\end{cases}
$$

Осталось заметить, что из равенств $(19),(10),(11)$ следует теорема 2. 


\section{СПИСОК ЦИТИРОВАННОЙ ЛИТЕРАТУРЫ}

[1] Краснов В.А. Отображение Альбанезе для вещественных алгебраических многообразий // Матем. заметки. 1982. Т. 32. № 3. С. 365-374.

[2] Nikulin V. V. On the Brauer group of real algebraic surfaces // Algebraic Geometry and Its Applications. Yaroslavl, 1992. P. 114-136.

[3] van Hamel J. Equivariant Borel-Moore homology and Poincaré duality for discrete transformation groups. Preprint.

[4] Краснов В.А. Когомологическая группа Брауэра вещественного алгебраического многообразия // Изв. РАН Сер. матем. 1996. Т. 60. № 5. С. 57-88.

[5] Краснов В.А. О группе Брауэра вещественной алгебраической поверхности // Матем. заметки. 1996. Т. 60. №6. С. 935-938.

[6] Sujatha R. Witt groups of real projective surfaces // Math. Ann. 1990. V. 288. P. 89-101.

[7] К раснов В.А. Этальные и эквивариантные когомологии вещественного алгебраического многообразия // Изв. РАН. Сер. матем. (в печати).

[8] Краснов В.А. Характеристические классы векторных расслоений на вешественном алгебраическом многообразии // Изв. АН СССР. Сер. матем. 1991. Т. 55. № 4. С. 716-746.

[9] Калинин И.О. Когомологические характеристики вещественных проективных гиперповерхностей // Алгебра и анализ. 1991. Т. 3. № 2. С. 91-110. 